\title{
The effect of various subject characteristics on plantar pressure pattern
}

\author{
Noël LW Keijsers ${ }^{1 *}$, Niki M Stolwijk', Jan-Willem K Louwerens ${ }^{2}$ \\ From 4th Congress of the International Foot and Ankle Biomechanics (i-FAB) Community \\ Busan, Korea. 8-11 April 2014
}

\section{Background}

Plantar pressure is highly influenced by many factors such as walking velocity, body weight, and age. The impact of these subject characteristics on plantar pressure is usually studied separately. However, many of these factors are interact with each other; for example walking velocity is negatively correlated with body weight and age. The purpose of this study is to investigate the effect of several subject characteristics in relation to plantar pressure pattern for a large group of subjects.

\section{Materials and methods}

Plantar pressure measurements of 589 subjects were used in this study. All subjects walked barefoot over a Foot Scan pressure plate (Rsscan International, Olen, Belgium) mounted on top of a force plate (Kistler Instruments, Switzerland) at their preferred walking speed. A total of five trials per foot were measured using a 3-step-protocol. Plantar pressure data was analyzed for each pixel by using the normalization method of Keijsers et. al. [1]. For each pixel, a multiple step forward linear regression analysis was used with mean pressure as dependent variable and the following subject characteristics as independent variables: body weight, contact time, age, body length, sex, foot progression angle, foot length, foot width, and side. Finally, the correlation coefficient of the full model for each pixel was calculated.

\section{Results}

The subject characteristics varied largely between subjects. The influence of each factor on the pressure of each pixel is shown in Figure 1. Body weight was the most important factor and was selected as parameter in $80.0 \%$ of the pixels. Body weight and walking velocity mainly have a positive effect on plantar pressure, whereas body length has a negative effect. The pressure under the heel, midfoot and distal part of the forefoot showed the highest correlation coefficient values with subject characteristics.

*Correspondence: n.keijsers@maartenskliniek.nl

'Department of Research, Sint Maartenskliniek, Nijmegen, the Netherlands

Full list of author information is available at the end of the article 


\section{Conclusions}

Subject characteristics and especially the body weight and foot size play an important role in plantar pressure. Multiple regression analysis or adding subject characteristics as covariates is recommended when differences in plantar pressure between groups are studied.

\section{Authors' details}

'Department of Research, Sint Maartenskliniek, Nijmegen, the Netherlands.

${ }^{2}$ Department of Orthopaedics, Sint Maartenskliniek, Nijmegen, the

Netherlands.

Published: 8 April 2014

\section{Reference}

1. Keijsers, et al: J. Biomech 2009, 42:87-90.

doi:10.1186/1757-1146-7-S1-A40

Cite this article as: Keijsers et al:: The effect of various subject

characteristics on plantar pressure pattern. Journal of Foot and Ankle

Research 2014 7(Suppl 1):A40. 